\title{
On convergence of generators of equilibrium dynamics of hopping particles to generator of a birth-and-death process in continuum*
}

\author{
E.Lytvynov, P.T.Polara \\ Department of Mathematics, Swansea University, Singleton Park, Swansea, SA2 8PP, U.K.
}

Received January 31, 2008

\begin{abstract}
We deal with the two following classes of equilibrium stochastic dynamics of infinite particle systems in continuum: hopping particles (also called Kawasaki dynamics), i.e., a dynamics where each particle randomly hops over the space, and birth-and-death process in continuum (or Glauber dynamics), i.e., a dynamics where there is no motion of particles, but rather particles die, or are born at random. We prove that a wide class of Glauber dynamics can be derived as a scaling limit of Kawasaki dynamics. More precisely, we prove the convergence of respective generators on a set of cylinder functions, in the $L^{2}$-norm with respect to the invariant measure of the processes. The latter measure is supposed to be a Gibbs measure corresponding to a potential of pair interaction, in the low activity-high temperature regime. Our result generalizes that of [Random. Oper. Stoch. Equa., 2007, 15, 105], which was proved for a special Glauber (Kawasaki, respectively) dynamics.
\end{abstract}

Key words: birth-and-death process, continuous system, Gibbs measure, hopping particles, scaling limit

PACS: $02.50 . E y, 02.50 . G a$

\section{Introduction}

This paper deals with two classes of equilibrium stochastic dynamics of infinite particle systems in continuum. Let $\Gamma$ denote the space of all locally finite subsets of $\mathbb{R}^{d}$. Such a space is called the configuration space (of an infinite particle system in continuum). Elements of $\Gamma$ are called configurations and each point of a configuration represents the position of a particle.

One can naturally define a $\sigma$-algebra on $\Gamma$, and then a probability measure on $\Gamma$ represents a random system of particles. A probability measure on $\Gamma$ is often referred to as a point process (see e.g. [9]). Configuration spaces and point processes are important tools in classical statistical mechanics of continuous systems. A central class of point processes which is studied therein is the class of Gibbs measures. Typically one deals with Gibbs measures that correspond to a potential of pair interaction.

An equilibrium stochastic dynamics in continuum is a Markov process on $\Gamma$ that has a point process $\mu$ (typically a Gibbs measure) as its invariant measure. One can distinguish three main classes of stochastic dynamics:

- diffusion processes, i.e., dynamics where each particle continuously moves in the space, see, e.g., $[2,4,12,16,18,20,24]$;

- birth-and-death processes in continuum (Glauber dynamics), i.e., dynamics where there is no motion of particles, but rather particles disappear (die) or appear (are born) at random, see, e.g., $[1,6,8,11,13,14,19,23]$;

- hopping particles (Kawasaki dynamics), i.e., dynamics where each particle randomly hops over the space [13].

*The first named author acknowledges the financial support of the Royal Society 2007/R2 Conference Grant. 
In order to profoundly understand these dynamics, it is important to see how they are related to each other. For example, in the recent paper [10], it was shown that a typical diffusion dynamics can be derived through a diffusive scaling limit of a corresponding Kawasaki dynamics.

In [3], it was proved that a special Glauber dynamics can be derived through a scaling limit of Kawasaki dynamics. Furthermore, it was conjectured in [3] that such a result holds, in fact, for a wide class of birth-and-death dynamics (dynamics of hopping particles, respectively), which are indexed by a parameter $s \in[0,1]$. (Note that the result of [3] corresponds to the choice of parameter $s=0$.)

The purpose of this work is to show that the conjecture of [3] is indeed true, at least for parameters $s \in[0,1 / 2]$. (In the case where $s \in(1 / 2,1]$, one needs to put additional, quite restrictive assumptions on the potential of pair interaction, and we will not treat this case in the present paper.) Thus, we show that the result of [3] is not a property of just one special Kawasaki (Glauber, respectively) dynamics, but rather represents a property which is common to many dynamics.

More specifically, we fix a class of cylinder functions on $\Gamma$, and prove that on this class of functions, the corresponding generators converge in the $L^{2}(\Gamma, \mu)$-space. Here, $\mu$ is a Gibbs measure in the low activity-high temperature regime, $\mu$ being invariant measure for all the processes under consideration. If one knows that the class of cylinder functions is a core for the limiting generator, then our result implies weak convergence of finite-dimensional distributions of the corresponding processes. Unfortunately, apart from a very special case [11], no result concerning a core for these generators is yet available.

The paper is organized as follows. In section 2, we briefly discuss Gibbs measures in the low activity-high temperature regime, and the corresponding correlation and Ursell functions. In section 3, we describe the classes of birth-and-death processes and of dynamics of hopping particles. In section 4 , we formulate and prove the result concerning the convergence of the generators.

The authors acknowledge numerous useful discussions with Dmirti Finkelshtein and Yuri Kondratiev.

\section{Gibbs measures in the low activity-high temperature regime}

The configuration space over $\mathbb{R}^{d}, d \in \mathbb{N}$, is defined by

$$
\Gamma:=\left\{\gamma \subset \mathbb{R}^{d}:|\gamma \cap \Lambda|<\infty \text { for each compact } \Lambda \subset \mathbb{R}^{d}\right\}
$$

where $|\cdot|$ denotes the cardinality of a set. One can identify any $\gamma \in \Gamma$ with the positive Radon measure $\sum_{x \in \gamma} \varepsilon_{x} \in \mathcal{M}\left(\mathbb{R}^{d}\right)$, where $\varepsilon_{x}$ is the Dirac measure with mass at $x, \sum_{x \in \varnothing} \varepsilon_{x}:=$ zero measure, and $\mathcal{M}\left(\mathbb{R}^{d}\right)$ stands for the set of all positive Radon measures on the Borel $\sigma$-algebra $\mathcal{B}\left(\mathbb{R}^{d}\right)$. The space $\Gamma$ can be endowed with the relative topology as a subset of the space $\mathcal{M}\left(\mathbb{R}^{d}\right)$ with the vague topology, i.e., the weakest topology on $\Gamma$ with respect to which all the maps

$$
\Gamma \ni \gamma \mapsto\langle f, \gamma\rangle:=\int_{\mathbb{R}^{d}} f(x) \gamma(\mathrm{d} x)=\sum_{x \in \gamma} f(x), \quad f \in C_{0}\left(\mathbb{R}^{d}\right)
$$

are continuous. Here, $C_{0}\left(\mathbb{R}^{d}\right)$ is the space of all continuous real-valued functions on $\mathbb{R}^{d}$ with compact support. We will denote by $\mathcal{B}(\Gamma)$ the Borel $\sigma$-algebra on $\Gamma$.

Let $\mu$ be a probability measure on $(\Gamma, \mathcal{B}(\Gamma))$. Assume that, for each $n \in \mathbb{N}$, there exists a nonnegative, measurable symmetric function $k_{\mu}^{(n)}$ on $\left(\mathbb{R}^{d}\right)^{n}$ such that, for any measurable symmetric function $f^{(n)}:\left(\mathbb{R}^{d}\right)^{n} \rightarrow[0,+\infty]$,

$$
\int_{\Gamma} \sum_{\left\{x_{1}, \ldots, x_{n}\right\} \subset \gamma} f^{(n)}\left(x_{1}, \ldots, x_{n}\right) \mu(\mathrm{d} \gamma)=\frac{1}{n !} \int_{\left(\mathbb{R}^{d}\right)^{n}} f^{(n)}\left(x_{1}, \ldots, x_{n}\right) k_{\mu}^{(n)}\left(x_{1}, \ldots, x_{n}\right) \mathrm{d} x_{1} \cdots \mathrm{d} x_{n}
$$

The functions $k_{\mu}^{(n)}$ are called correlation functions of the measure $\mu$. If there exists a constant $\xi>0$ such that

$$
\forall\left(x_{1}, \ldots, x_{n}\right) \in\left(\mathbb{R}^{d}\right)^{n}: \quad k_{\mu}^{(n)}\left(x_{1}, \ldots, x_{n}\right) \leqslant \xi^{n},
$$


then we say that the correlation functions $k_{\mu}^{(n)}$ satisfy the Ruelle bound.

The following lemma gives a characterization of the correlation functions in terms of the Laplace transform of a given point process, see, e.g., [10]

Lemma 1. Let $\mu$ be a probability measure on $(\Gamma, \mathcal{B}(\Gamma))$ which satisfies the Ruelle bound (1). Let $f: \mathbb{R}^{d} \rightarrow \mathbb{R}$ be a measurable function which is bounded outside a compact set $\Lambda \subset \mathbb{R}^{d}$ and such that $\mathrm{e}^{f}-1 \in L^{1}\left(\mathbb{R}^{d}, \mathrm{~d} x\right)$. Then for $\mu$-a.a. $\gamma \in \Gamma,\langle|f|, \gamma\rangle<\infty$ and

$$
\int_{\Gamma} \mathrm{e}^{\langle f, \gamma\rangle} \mu(\mathrm{d} \gamma)=1+\sum_{n=1}^{\infty} \frac{1}{n !} \int_{\left(\mathbb{R}^{d}\right)^{n}}\left(\mathrm{e}^{f\left(x_{1}\right)}-1\right) \cdots\left(\mathrm{e}^{f\left(x_{n}\right)}-1\right) k_{\mu}^{(n)}\left(x_{1}, \ldots, x_{n}\right) \mathrm{d} x_{1} \cdots \mathrm{d} x_{n} .
$$

Remark 1. Note that if $f: \mathbb{R}^{d} \rightarrow \mathbb{R}$ is bounded outside a compact set $\Lambda \subset \mathbb{R}^{d}$ and if, furthermore, $f$ is bounded from above on the whole $\mathbb{R}^{d}$, then the condition $\mathrm{e}^{f}-1 \in L^{1}\left(\mathbb{R}^{d}, \mathrm{~d} x\right)$ is equivalent to $f \in L^{1}\left(\Lambda^{c}, \mathrm{~d} x\right)$.

Via a recursion formula, one can transform the correlation functions $k_{\mu}^{(n)}$ into the Ursell functions $u_{\mu}^{(n)}$ and vice versa, see, e.g., [22]. Their relation is given by

$$
k_{\mu}(\eta)=\sum u_{\mu}\left(\eta_{1}\right) \cdots u_{\mu}\left(\eta_{j}\right), \quad \eta \in \Gamma_{0}, \eta \neq \varnothing
$$

where

$$
\Gamma_{0}:=\{\gamma \in \Gamma:|\gamma|<\infty\}
$$

for any $\eta=\left\{x_{1}, \ldots, x_{n}\right\} \in \Gamma_{0}$

$$
k_{\mu}(\eta):=k_{\mu}^{(n)}\left(x_{1}, \ldots, x_{n}\right), \quad u_{\mu}(\eta):=u_{\mu}^{(n)}\left(x_{1}, \ldots, x_{n}\right),
$$

and the summation in (2) is over all partitions of the set $\eta$ into nonempty mutually disjoint subsets $\eta_{1}, \ldots, \eta_{j} \subset \eta$ such that $\eta_{1} \cup \cdots \cup \eta_{j}=\eta, j \in \mathbb{N}$. Note that if the correlation functions $\left(k_{\mu}^{(n)}\right)_{n=1}^{\infty}$ are translation invariant, i.e., for each $a \in \mathbb{R}^{d}$

$$
k_{\mu}^{(n)}\left(x_{1}, \ldots, x_{n}\right)=k_{\mu}^{(n)}\left(x_{1}+a, \ldots, x_{n}+a\right), \quad\left(x_{1}, \ldots, x_{n}\right) \in\left(\mathbb{R}^{d}\right)^{n},
$$

then so are the Ursell functions $\left(u_{\mu}^{(n)}\right)_{n=1}^{\infty}$.

A pair potential is a Borel-measurable function $\phi: \mathbb{R}^{d} \rightarrow \mathbb{R} \cup\{+\infty\}$ such that $\phi(-x)=\phi(x) \in \mathbb{R}$ for all $x \in \mathbb{R}^{d} \backslash\{0\}$. For $\gamma \in \Gamma$ and $x \in \mathbb{R}^{d} \backslash \gamma$, we define a relative energy of interaction between a particle at $x$ and the configuration $\gamma$ as follows:

$$
E(x, \gamma):= \begin{cases}\sum_{y \in \gamma} \phi(x-y), & \text { if } \sum_{y \in \gamma}|\phi(x-y)|<+\infty, \\ +\infty, & \text { otherwise }\end{cases}
$$

A probability measure $\mu$ on $(\Gamma, \mathcal{B}(\Gamma))$ is called a (grand canonical) Gibbs measure corresponding to the pair potential $\phi$ and activity $z>0$ if it satisfies the Georgii-Nguyen-Zessin identity ([17, Theorem 2]):

$$
\int_{\Gamma} \mu(\mathrm{d} \gamma) \int_{\mathbb{R}^{d}} \gamma(\mathrm{d} x) F(\gamma, x)=\int_{\Gamma} \mu(\mathrm{d} \gamma) \int_{\mathbb{R}^{d}} z \mathrm{~d} x \exp [-E(x, \gamma)] F(\gamma \cup x, x)
$$

for any measurable function $F: \Gamma \times \mathbb{R}^{d} \rightarrow[0,+\infty]$. Here and below, for simplicity of notations, we just write $x$ instead of $\{x\}$. We denote the set of all such measures $\mu$ by $\mathcal{G}(z, \phi)$.

As a straightforward corollary of the Georgii-Nguyen-Zessin identity (3), we get the following equality:

$$
\begin{aligned}
\int_{\Gamma} \mu(\mathrm{d} \gamma) \int_{\mathbb{R}^{d}} \gamma\left(\mathrm{d} x_{1}\right) \int_{\mathbb{R}^{d}} \gamma\left(\mathrm{d} x_{2}\right) F\left(\gamma, x_{1}, x_{2}\right) \\
=\int_{\Gamma} \mu(\mathrm{d} \gamma) \int_{\mathbb{R}^{d}} z \mathrm{~d} x_{1} \int_{\mathbb{R}^{d}} z \mathrm{~d} x_{2} \exp \left[-E\left(x_{1}, \gamma\right)-E\left(x_{2}, \gamma\right)-\phi\left(x_{1}-x_{2}\right)\right] \\
\quad \times F\left(\gamma \cup\left\{x_{1}, x_{2}\right\}, x_{1}, x_{2}\right)+\int_{\Gamma} \mu(\mathrm{d} \gamma) \int_{\mathbb{R}^{d}} z \mathrm{~d} x \exp [-E(x, \gamma)] F(\gamma \cup x, x, x)
\end{aligned}
$$


for any measurable function $F: \Gamma \times \mathbb{R}^{d} \times \mathbb{R}^{d} \rightarrow[0,+\infty]$.

Let us formulate the conditions on the pair potential $\phi$.

(S) (Stability) There exists $B \geqslant 0$ such that, for any $\gamma \in \Gamma_{0}$,

$$
\sum_{\{x, y\} \subset \gamma} \phi(x-y) \geqslant-B|\gamma| .
$$

In particular, condition (S) implies that $\phi(x) \geqslant-2 B, x \in \mathbb{R}^{d}$.

(LA-HT) (Low activity-high temperature regime) We have:

$$
\int_{\mathbb{R}^{d}}\left|\mathrm{e}^{-\phi(x)}-1\right| z \mathrm{~d} x<\left(2 \mathrm{e}^{1+2 B}\right)^{-1},
$$

where $B$ is as in $(\mathrm{S})$.

The following classical theorem is due to Ruelle $[21,22]$.

Theorem 1. Assume that (S) and (LAHT) are satisfied. Then there exists $\mu \in \mathcal{G}(z, \phi)$ which has the following properties:

a) $\mu$ has correlation functions $\left(k_{\mu}^{(n)}\right)_{n=1}^{\infty}$, which are translation invariant and satisfy the Ruelle bound (1);

b) For each $n \geqslant 2$, we have $u_{\mu}^{(n)}(0, \cdot, \cdot, \ldots, \cdot) \in L^{1}\left(\mathbb{R}^{d(n-1)}, \mathrm{d} x_{1} \cdots \mathrm{d} x_{n-1}\right)$, where $u_{\mu}^{(n)}(0, \cdot, \cdot, \ldots, \cdot)$ is considered as a function of $n-1$ variables.

In what follows, we will assume that $(\mathrm{S})$ and (LAHT) are satisfied, and we will keep the measure $\mu$ from Theorem 1 fixed.

\section{Equilibrium birth-and-death (Glauber) dynamics and hopping particles' (Kawasaki) dynamics}

In what follows, we will also assume that $\phi$ is bounded outside some ball in $\mathbb{R}^{d}$. Note that then (see e.g. [13])

$$
E(x, \gamma)=\sum_{y \in \gamma} \phi(x-y)
$$

for $\mathrm{d} x \mu(\mathrm{d} \gamma)$-a.a. $x \in \mathbb{R}^{d}$ and $\gamma \in \Gamma$ and

$$
E(x, \gamma \backslash x)=\sum_{y \in \gamma \backslash x} \phi(x-y)
$$

for $\mu$-a.a. $\gamma \in \Gamma$ and all $x \in \gamma$.

We fix a parameter $s \in[0,1 / 2]$. We introduce the set $\mathcal{F} C_{b}\left(C_{0}\left(\mathbb{R}^{d}\right), \Gamma\right)$ of all functions of the form

$$
\Gamma \ni \gamma \mapsto F(\gamma)=g\left(\left\langle f_{1}, \gamma\right\rangle, \ldots,\left\langle f_{N}, \gamma\right\rangle\right),
$$

where $N \in \mathbb{N}, f_{1}, \ldots, f_{N} \in C_{0}\left(\mathbb{R}^{d}\right)$, and $g \in C_{b}\left(\mathbb{R}^{N}\right)$, where $C_{b}\left(\mathbb{R}^{N}\right)$ denotes the set of all continuous bounded functions on $\mathbb{R}^{N}$. For each function $F: \Gamma \rightarrow \mathbb{R}, \gamma \in \Gamma$, and $x, y \in \mathbb{R}^{d}$, we denote

$$
\begin{gathered}
\left(D_{x}^{-} F\right)(\gamma):=F(\gamma \backslash x)-F(\gamma), \\
\left(D_{x y}^{-+} F\right)(\gamma):=F(\gamma \backslash x \cup y)-F(\gamma) .
\end{gathered}
$$

We fix a bounded function $a: \mathbb{R}^{d} \rightarrow[0,+\infty)$ such that $a(-x)=a(x), x \in \mathbb{R}^{d}$, and $a \in$ $L^{1}\left(\mathbb{R}^{d}, d x\right)$. We define bilinear forms

$$
\begin{aligned}
\mathcal{E}_{G}(F, G)= & \int_{\Gamma} \mu(\mathrm{d} \gamma) \int_{\mathbb{R}^{d}} \gamma(\mathrm{d} x) \exp [s E(x, \gamma \backslash x)]\left(D_{x}^{-} F\right)(\gamma)\left(D_{x}^{-} G\right)(\gamma), \\
\mathcal{E}_{K}(F, G)= & \frac{1}{2} \int_{\Gamma} \mu(\mathrm{d} \gamma) \int_{\mathbb{R}^{d}} \gamma(\mathrm{d} x) \int_{\mathbb{R}^{d}} \mathrm{~d} y a(x-y) \\
& \times \exp [s E(x, \gamma \backslash x)-(1-s) E(y, \gamma \backslash x)]\left(D_{x y}^{-+} F\right)(\gamma)\left(D_{x y}^{-+} G\right)(\gamma),
\end{aligned}
$$


where $F, G \in \mathcal{F} C_{b}\left(C_{0}\left(\mathbb{R}^{d}\right), \Gamma\right)$. As we will see below, $\mathcal{E}_{G}$ corresponds to a Glauber dynamics and $\mathcal{E}_{K}$ corresponds to a Kawasaki dynamics.

The next theorem follows from [13].

Theorem 2. i) The bilinear forms $\left(\mathcal{E}_{G}, \mathcal{F} C_{b}\left(C_{0}\left(\mathbb{R}^{d}\right), \Gamma\right)\right)$ and $\left(\mathcal{E}_{K}, \mathcal{F} C_{b}\left(C_{0}\left(\mathbb{R}^{d}\right), \Gamma\right)\right)$ are closable on $L^{2}(\Gamma, \mu)$ and their closures are denoted by $\left(\mathcal{E}_{G}, D\left(\mathcal{E}_{G}\right)\right)$ and $\left(\mathcal{E}_{K}, D\left(\mathcal{E}_{K}\right)\right)$, respectively.

ii) Denote by $\left(H_{G}, D\left(H_{G}\right)\right)$ and $\left(H_{K}, D\left(H_{K}\right)\right)$ the generators of $\left(\mathcal{E}_{G}, D\left(\mathcal{E}_{G}\right)\right)$ and $\left(\mathcal{E}_{K}, D\left(\mathcal{E}_{K}\right)\right)$, respectively. Then $\mathcal{F} C_{b}\left(C_{0}\left(\mathbb{R}^{d}\right), \Gamma\right) \subset D\left(H_{G}\right) \cap D\left(H_{K}\right)$, and for any $F \in \mathcal{F} C_{b}\left(C_{0}\left(\mathbb{R}^{d}\right), \Gamma\right)$,

$$
\begin{aligned}
& \left(H_{G} F\right)(\gamma)=-\int_{\mathbb{R}^{d}} \gamma(\mathrm{d} x) \exp [s E(x, \gamma \backslash x)]\left(D_{x}^{-} F\right)(\gamma)-\int_{\mathbb{R}^{d}} z \mathrm{~d} x \exp [(s-1) E(x, \gamma)]\left(D_{x}^{+} F\right)(\gamma), \\
& \left(H_{K} F\right)(\gamma)=-\int_{\mathbb{R}^{d}} \gamma(\mathrm{d} x) \int_{\mathbb{R}^{d}} \mathrm{~d} y a(x-y) \exp [s E(x, \gamma \backslash x)+(s-1) E(y, \gamma \backslash x)]\left(D_{x y}^{-+} F\right)(\gamma)
\end{aligned}
$$

iii) Let $\sharp:=G, K$. There exists a conservative Hunt process

$$
\mathbf{M}^{\sharp}=\left(\mathbf{\Omega}^{\sharp}, \mathbf{F}^{\sharp},\left(\mathbf{F}_{t}^{\sharp}\right)_{t \geqslant 0},\left(\boldsymbol{\Theta}_{t}^{\sharp}\right)_{t \geqslant 0},\left(\mathbf{X}^{\sharp}(t)_{t \geqslant 0},\left(\mathbf{P}_{\gamma}^{\sharp}\right)_{\gamma \in \Gamma}\right)\right.
$$

on $\Gamma$ (see, e.g., $\left[15\right.$, p. 92]) which is properly associated with $\left(\mathcal{E}_{\sharp}, D\left(\mathcal{E}_{\sharp}\right)\right)$, i.e., for all ( $\mu$-versions of) $F \in L^{2}(\Gamma, \mu)$ and all $t>0$ the function

$$
\Gamma \ni \gamma \mapsto\left(p_{t}^{\sharp} F\right)(\gamma):=\int_{\Omega^{\sharp}} F\left(\mathbf{X}^{\sharp}(t)\right) \mathrm{d} \mathbf{P}_{\gamma}^{\sharp}
$$

is an $\mathcal{E}_{\sharp}$-quasi-continuous version of $\exp \left[-t H_{\sharp}\right] F . \mathbf{M}^{\sharp}$ is up to $\mu$-equivalence unique (cf. [15, Chap. IV, Sect. 6]). In particular, $\mathbf{M}^{\sharp}$ has $\mu$ as invariant measure.

Remark 2. In Theorem $2, \mathbf{M}^{\sharp}$ can be taken canonical, i.e., $\boldsymbol{\Omega}^{\sharp}$ is the set $D([0,+\infty), \Gamma)$ of all cádlág functions $\omega:[0,+\infty) \rightarrow \Gamma$ (i.e., $\omega$ is right continuous on $[0,+\infty)$ and has left limits on $(0,+\infty)) ; \mathbf{X}^{\sharp}(t)(\omega)=\omega(t), t \geqslant 0, \omega \in \Omega^{\sharp} ;\left(\mathbf{F}_{t}^{\sharp}\right)_{t \geqslant 0}$, together with $\mathbf{F}^{\sharp}$, is the corresponding minimum completed admissible family (cf. [5, Section 4.1]); $\Theta_{t}^{\sharp}, t \geqslant 0$, are the corresponding natural time shifts.

It follows from (5) that $H_{G}$ is (at least heuristically) the generator of a birth-and-death process, in which the factor $\exp [s E(x, \gamma \backslash x)]$ describes the rate at which particle $x$ of the configuration $\gamma$ dies, whereas the factor $\exp [(s-1) E(x, \gamma)]$ describes the rate at which, given a configuration $\gamma$, a new particle is born at $x$. We see that particles tend to die in high energy regions, i.e., if $E(x, \gamma \backslash x)$ is high, and they tend to be born al low energy regions, i.e., if $E(x, \gamma)$ is low.

Next, by (6), $H_{K}$ is (again at least heuristically) the generator of a hopping particle dynamics, in which the factor

$$
\exp [s E(x, \gamma \backslash x)+(s-1) E(y, \gamma \backslash x)]
$$

describes the rate at which a particle $x$ of configuration $\gamma$ hops to $y$. We see that this rate is high if the relative energy of interaction between $x$ and the rest of the configuration, $\gamma \backslash x$, is high, whereas the relative energy of interaction between $y$ and $\gamma \backslash x$ is low, i.e., particles tend to hop from high energy regions to low energy regions.

\section{Scaling limit}

In this section, we will show that the birth-and-death dynamics considered in section 3 can be treated as a limiting dynamics of hopping particles. In other words, we will perform a scaling of Kawasaki dynamics which will lead to the Glauber dynamics. We will only discuss this convergence at the level of convergence of the generators on an appropriate set of cylinder functions. In fact, such a convergence implies a weak convergence of finite-dimensional distributions of corresponding equilibrium processes if the set of test functions additionally forms a core for the limiting generator. However, in the general case, no core of this generator is yet known and this is an open, important 
problem, which we hope to return to in our future research. Our results will hold for all $s \in[0,1 / 2]$ (see section 3). They will generalize Theorem 4.1 in [3], which was proved in the special case $s=0$, and confirm the conjecture formulated in section 6 of that paper.

So, let us consider the following scaling of the Kawasaki dynamics (for a fixed $s \in[0,1 / 2]$ ). Recall that, for each bounded function $a: \mathbb{R}^{d} \rightarrow \mathbb{R}$ such that $a(x) \geqslant 0, a \in L^{1}\left(\mathbb{R}^{d}, \mathrm{~d} x\right)$, and $a(-x)=a(x)$ for all $x \in \mathbb{R}^{d}$, we have constructed the corresponding generator of the Kawasaki dynamics. We now fix an arbitrary $\varepsilon>0$ and define a function $a_{\varepsilon}: \mathbb{R}^{d} \rightarrow \mathbb{R}$ by

$$
a_{\varepsilon}(x)=\varepsilon^{d} a(\varepsilon x), \quad x \in \mathbb{R}^{d} .
$$

Note that

$$
\int_{\mathbb{R}^{d}} a_{\varepsilon}(x) \mathrm{d} x=\int_{\mathbb{R}^{d}} a(x) \mathrm{d} x .
$$

By the properties of the function $a$, we evidently have that the function $a_{\varepsilon}$ is also bounded, satisfies $a_{\varepsilon}(x) \geqslant 0$, for all $x \in \mathbb{R}^{d}, a_{\varepsilon} \in L^{1}\left(\mathbb{R}^{d}, d x\right)$, and $a_{\varepsilon}(-x)=a_{\varepsilon}(x)$ for all $x \in \mathbb{R}^{d}$. Hence, we can construct the Kawasaki generator which corresponds to the function $a_{\varepsilon}$. It is convenient for us to denote this generator by $\left(H_{\varepsilon}, D\left(H_{\varepsilon}\right)\right)$. We will also denote the generator of the Glauber dynamics by $\left(H_{0}, D\left(H_{0}\right)\right)$. We first need the following lemma, whose proof is completely analogous to the proof of Lemma 4.1 in [3].

Lemma 2. For any $\varepsilon \geqslant 0$ and any $\varphi \in C_{0}\left(\mathbb{R}^{d}\right)$, the function $F(\gamma):=\mathrm{e}^{\langle\varphi, \gamma\rangle}$ belongs to $D\left(H_{\varepsilon}\right)$ and the action of $H_{\varepsilon}$ on $F$ is given by the right hand side of formula (6) for $\varepsilon>0$ (with a replaced by $\left.a_{\varepsilon}\right)$, respectively by the right hand side of (5) for $\varepsilon=0$.

Remark 3. For each $\varepsilon \geqslant 0$, denote by $\left(\mathcal{E}_{\varepsilon}, D\left(\mathcal{E}_{\varepsilon}\right)\right)$ the Dirichlet form with the generator $\left(H_{\varepsilon}, D\left(H_{\varepsilon}\right)\right)$. It can be easily proved that the set $\left\{\exp [\langle\varphi, \cdot\rangle]: \varphi \in C_{0}\left(\mathbb{R}^{d}\right)\right\}$ is dense in the Hilbert space $D\left(\mathcal{E}_{\varepsilon}\right)$ equipped with inner product $(F, G)_{D\left(\mathcal{E}_{\epsilon}\right)}:=\mathcal{E}(F, G)+(F, G)_{L^{2}(\Gamma, \mu)}$.

We have

$$
\begin{aligned}
\left(D_{x y}^{-+} F\right)(\gamma) & =F(\gamma \backslash x \cup y)-F(\gamma)=-F(\gamma)+F(\gamma \backslash x)-F(\gamma \backslash x)+F(\gamma \backslash x \cup y) \\
& =\left(D_{x}^{-} F\right)(\gamma)+\left(D_{y}^{+} F\right)(\gamma \backslash x) .
\end{aligned}
$$

So, we may rewrite the action of $H_{\varepsilon}$ for $\varepsilon>0$ as follows:

$$
H_{\varepsilon}:=H_{\varepsilon}^{+}+H_{\varepsilon}^{-},
$$

where

$$
\left(H_{\varepsilon}^{-} F\right)(\gamma)=-\int_{\mathbb{R}^{d}} \gamma(\mathrm{d} x)\left(D_{x}^{-} F\right)(\gamma) \int_{\mathbb{R}^{d}} \mathrm{~d} y a_{\varepsilon}(x-y) \exp [s E(x, \gamma \backslash x)+(s-1) E(y, \gamma \backslash x)]
$$

and

$$
\left(H_{\varepsilon}^{+} F\right)(\gamma)=-\int_{\mathbb{R}^{d}} \gamma(\mathrm{d} x) \int_{\mathbb{R}^{d}} \mathrm{~d} y a_{\varepsilon}(x-y) \exp [s E(x, \gamma \backslash x)+(s-1) E(y, \gamma \backslash x)]\left(D_{y}^{+} F\right)(\gamma \backslash x) .
$$

We can also rewrite

$$
H_{0}:=H_{0}^{+}+H_{0}^{-} \text {, }
$$

where

$$
\left(H_{0}^{-} F\right)(\gamma)=-\int_{\mathbb{R}^{d}} \gamma(\mathrm{d} x) \exp [s E(x, \gamma \backslash x)]\left(D_{x}^{-} F\right)(\gamma)
$$

and

$$
\left(H_{0}^{+} F\right)(\gamma)=-\int_{\mathbb{R}^{d}} z \mathrm{~d} x \exp [-(1-s) E(x, \gamma)]\left(D_{x}^{+} F\right)(\gamma)
$$


Theorem 3. Let $s \in[0,1 / 2]$ be fixed. Assume that the pair potential $\phi$ and activity $z>0$ satisfy conditions $(\mathrm{S})$ and $(\mathrm{LA}-\mathrm{HT})$. Assume that $\phi$ is bounded outside some compact set in $\mathbb{R}^{d}$. Assume also that

$$
\phi(x) \rightarrow 0 \text { as }|x| \rightarrow \infty .
$$

Let $\mu$ be the Gibbs measure from $\mathcal{G}(z, \phi)$ as in Theorem 1. Assume that the function a is chosen so that

$$
\int_{\mathbb{R}^{d}} a(x) \mathrm{d} x=\left(\int_{\Gamma} \exp \left[(s-1) \sum_{u \in \gamma} \phi(u)\right] \mu(\mathrm{d} \gamma)\right)^{-1}
$$

Then, for any $\varphi \in C_{0}\left(\mathbb{R}^{d}\right)$,

$$
H_{\varepsilon}^{ \pm} \mathrm{e}^{\langle\varphi, \cdot\rangle} \rightarrow H_{0}^{ \pm} \mathrm{e}^{\langle\varphi, \cdot\rangle} \text { in } L^{2}(\Gamma, \mu) \text { as } \varepsilon \rightarrow 0,
$$

so that

$$
H_{\varepsilon} \mathrm{e}^{\langle\varphi, \cdot\rangle} \rightarrow H_{0} \mathrm{e}^{\langle\varphi, \cdot\rangle} \text { in } L^{2}(\Gamma, \mu) \text { as } \varepsilon \rightarrow 0 .
$$

Remark 4. In fact, condition (7) can be omitted, and instead one can use the fact that $\phi$ is an integrable function outside a compact set in $\mathbb{R}^{d}$ (compare with [3]). However, in any reasonable application, the potential $\phi$ does satisfy condition (7).

Remark 5. Note that the integral on the right hand side of (8) is well defined and finite due to Lemma 1, see also Remark 1

Proof. We first need the following lemma, which generalizes Lemma 4.2 in [3].

Lemma 3. Let a function $\psi: \mathbb{R}^{d} \rightarrow \mathbb{R}$ be such that $\mathrm{e}^{\psi}-1$ is bounded and integrable. Suppose that $A \geqslant 0, B \geqslant 0, x_{1}, x_{2}, y_{1}, y_{2} \in \mathbb{R}^{d}$ and $x_{1} \neq y_{1}$. Then

$$
\begin{aligned}
\int_{\Gamma} \exp [-A E & \left.\left(\frac{x_{1}}{\varepsilon}+x_{2}, \gamma\right)-B E\left(\frac{y_{1}}{\varepsilon}+y_{2}, \gamma\right)+\langle\psi, \gamma\rangle\right] \mu(\mathrm{d} \gamma) \\
& \rightarrow \int_{\Gamma} \exp \left[-A \sum_{u \in \gamma} \phi(u)\right] \mu(\mathrm{d} \gamma) \int_{\Gamma} \exp \left[-B \sum_{u \in \gamma} \phi(u)\right] \mu(\mathrm{d} \gamma) \int_{\Gamma} \exp [\langle\psi, \gamma\rangle] \mu(\mathrm{d} \gamma)
\end{aligned}
$$

as $\varepsilon \rightarrow 0$.

Proof. By Lemma 1,

$$
\begin{aligned}
\int_{\Gamma} \exp [- & \left.A E\left(\left(x_{1} / \varepsilon\right)+x_{2}, \gamma\right)-B E\left(\left(y_{1} / \varepsilon\right)+y_{2}, \gamma\right)+\langle\psi, \gamma\rangle\right] \mu(\mathrm{d} \gamma) \\
= & 1+\sum_{n=1}^{\infty} \frac{1}{n !} \int_{\left(\mathbb{R}^{d}\right)^{n}}(\exp [-A \phi(\cdot-x(\varepsilon))-B \phi(\cdot-y(\varepsilon))+\psi(\cdot)]-1)^{\otimes}\left(u_{1}, \ldots, u_{n}\right) \\
& \times k_{\mu}^{(n)}\left(u_{1}, \ldots, u_{n}\right) \mathrm{d} u_{1} \cdots \mathrm{d} u_{n}
\end{aligned}
$$

where $x(\varepsilon):=\left(x_{1} / \varepsilon\right)+x_{2}, y(\varepsilon):=\left(y_{1} / \varepsilon\right)+y_{2}$.

Using the Ruelle bound, semi-boundedness of $\phi$ from below and the integrability of $\phi$ outside a compact set, we conclude from the dominated convergence theorem that, in order to find the limit of the right hand side of (9) as $\varepsilon \rightarrow 0$, it suffices to find the limit of each term

$$
\begin{aligned}
C_{\varepsilon}^{(n)}:= & \int_{\left(\mathbb{R}^{d}\right)^{n}}(\exp [-A \phi(\cdot-x(\varepsilon))-B \phi(\cdot-y(\varepsilon))+\psi(\cdot)]-1)^{\otimes n}\left(u_{1}, \ldots, u_{n}\right) \\
& \times k_{\mu}^{(n)}\left(u_{1}, \ldots, u_{n}\right) \mathrm{d} u_{1} \cdots \mathrm{d} u_{n} \\
= & \sum_{n_{1}+n_{2}+n_{3}=n}\left(\begin{array}{c}
n \\
n_{1} n_{2} n_{3}
\end{array}\right) \int_{\left(\mathbb{R}^{d}\right)^{n}}\left(f_{1, \varepsilon}^{\otimes n_{1}} \otimes f_{2, \varepsilon}^{\otimes n_{2}} \otimes f_{3, \varepsilon}^{\otimes n_{3}}\right)\left(u_{1}, \ldots, u_{n}\right) \\
& \times k_{\mu}^{(n)}\left(u_{1}, \ldots, u_{n}\right) \mathrm{d} u_{1} \cdots \mathrm{d} u_{n}
\end{aligned}
$$


where

$$
\begin{aligned}
f_{1, \varepsilon}(u) & :=(\exp [-\psi(u)]-1) \exp [-A \phi(u-x(\varepsilon))-B \phi(u-y(\varepsilon))], \\
f_{2, \varepsilon}(u) & :=(\exp [-A \phi(u-x(\varepsilon))]-1) \exp [-B \phi(u-y(\varepsilon))], \\
f_{3, \varepsilon}(u) & :=\exp [-B \phi(u-y(\varepsilon))]-1, \quad u \in \mathbb{R} .
\end{aligned}
$$

Using the definition of Ursell functions, we see that

$$
\begin{aligned}
\int_{\left(\mathbb{R}^{d}\right)^{n}}\left(f_{1, \varepsilon}^{\otimes n_{1}} \otimes f_{2, \varepsilon}^{\otimes n_{2}} \otimes f_{3, \varepsilon}^{\otimes n_{3}}\right)\left(u_{1}, \ldots, u_{n}\right) k_{\mu}^{(n)}\left(u_{1}, \ldots, u_{n}\right) \mathrm{d} u_{1} \cdots \mathrm{d} u_{n} \\
\quad=\sum \int_{\left(\mathbb{R}^{d}\right)^{n}}\left(f_{1, \varepsilon}^{\otimes n_{1}} \otimes f_{2, \varepsilon}^{\otimes n_{2}} \otimes f_{3, \varepsilon}^{\otimes n_{3}}\right)\left(u_{1}, \ldots, u_{n}\right) u_{\mu}\left(\eta_{1}\right) \cdots u_{\mu}\left(\eta_{j}\right) \mathrm{d} u_{1} \cdots \mathrm{d} u_{n},
\end{aligned}
$$

where the summation is over all partitions $\left\{\eta_{1}, \ldots, \eta_{j}\right\}$ of $\eta=\left\{u_{1}, \ldots, u_{n}\right\}$. We now have to distinguish the three following cases.

Case 1: Each element $\eta_{i}$ of the partition is either a subset of $\left\{u_{1}, \ldots, u_{n_{1}}\right\}$, or a subset of $\left\{u_{n_{1}+1}, \ldots, u_{n_{1}+n_{2}}\right\}$, or a subset of $\left\{u_{n_{1}+n_{2}+1}, \ldots, u_{n}\right\}$. Set

$$
\begin{gathered}
u_{i}^{\prime}=u_{i}-x(\varepsilon), \quad i=n_{1}+1, \ldots, n_{2}, \\
u_{i}^{\prime}=u_{i}-y(\varepsilon), \quad i=n_{2}+1, \ldots, n .
\end{gathered}
$$

Then using the translation invariance of the Ursell functions, we get that the corresponding term is equal to

$$
\int_{\left(\mathbb{R}^{d}\right)^{n}}\left(f_{1, \varepsilon}^{\otimes n_{1}} \otimes g_{2, \varepsilon}^{\otimes n_{2}} \otimes g_{3, \varepsilon}^{\otimes n_{3}}\right)\left(u_{1}, \ldots, u_{n}\right) u_{\mu}\left(\eta_{1}\right) \cdots u_{\mu}\left(\eta_{j}\right) \mathrm{d} u_{1} \cdots \mathrm{d} u_{n}
$$

where

$$
\begin{aligned}
& g_{2, \varepsilon}(u):=(\exp [-A \phi(u)]-1) \exp \left[-B \phi\left(u+\left(\left(x_{1}-y_{1}\right) / \varepsilon\right)+x_{2}-y_{2}\right)\right], \\
& g_{3, \varepsilon}(u):=\exp [-B \phi(u)]-1, \quad u \in \mathbb{R} .
\end{aligned}
$$

Note that $x_{1}-y_{1} \neq 0$ and so for any fixed $u$ (and $x_{2}, y_{2}$ ), we have

$$
\left|u+\left(\left(x_{1}-y_{1}\right) / \varepsilon\right)+x_{2}-y_{2}\right| \rightarrow+\infty \quad \text { as } \varepsilon \rightarrow 0 .
$$

By (7) and the dominated convergence theorem, we therefore have that (11) converges to

$$
\begin{aligned}
& \int_{\left(\mathbb{R}^{d}\right)^{n}}(\exp [-\psi(\cdot)]-1)^{\otimes n_{1}} \otimes(\exp [-A \phi(\cdot)]-1)^{\otimes n_{2}} \\
& \otimes(\exp [-B \phi(\cdot)]-1)^{\otimes n_{3}}\left(u_{1}, \ldots, u_{n}\right) u_{\mu}\left(\eta_{1}\right) \cdots u_{\mu}\left(\eta_{j}\right) \mathrm{d} u_{1} \cdots \mathrm{d} u_{n} .
\end{aligned}
$$

Case 2: There is an element of the partition which has non-empty intersections with both sets $\left\{u_{1}, \ldots, u_{n_{1}}\right\}$ and $\left\{u_{n_{1}+1}, \ldots, u_{n}\right\}$.

Using Theorem 1 , we have that, for each $n \in \mathbb{N}$,

$$
U_{\mu}^{(n+1)} \in L^{1}\left(\left(\mathbb{R}^{d}\right)^{n}, \mathrm{~d} x_{1} \cdots \mathrm{d} x_{n}\right),
$$

where

$$
U_{\mu}^{(n+1)}\left(x_{1}, \ldots, x_{n}\right):=u_{\mu}^{(n+1)}\left(x_{1}, \ldots, x_{n}, 0\right), \quad\left(x_{1}, \ldots, x_{n}\right) \in\left(\mathbb{R}^{d}\right)^{n} .
$$

Consider the integral

$$
\begin{aligned}
\int_{\left(\mathbb{R}^{d}\right)^{k}}\left(\exp \left[-\psi\left(u_{1}\right)\right]-1\right) u_{\mu}^{(k)}\left(u_{1}, \ldots, u_{k}\right) \mathrm{d} u_{1} \cdots \mathrm{d} u_{k} \\
\quad=\int_{\left(\mathbb{R}^{d}\right)^{k}}\left(\exp \left[-\psi\left(u_{1}\right)\right]-1\right) u_{\mu}^{(k)}\left(0, u_{2}-u_{1}, u_{3}-u_{1}, \ldots, u_{k}-u_{1}\right) \mathrm{d} u_{1} \cdots \mathrm{d} u_{k},
\end{aligned}
$$


where we used translation invariance of Ursell function. By changing variables $u_{1}^{\prime}=u_{1}, u_{2}^{\prime}=$ $u_{2}-u_{1}, \ldots, u_{k}^{\prime}=u_{k}-u_{1}$, we continue as follows:

$$
\begin{aligned}
& =\int_{\left(\mathbb{R}^{d}\right)^{k}}\left(\exp \left[-\psi\left(u_{1}^{\prime}\right)\right]-1\right) u_{\mu}^{(k)}\left(0, u_{2}^{\prime}, u_{3}^{\prime}, \ldots, u_{k}^{\prime}\right) \mathrm{d} u_{1}^{\prime} \cdots \mathrm{d} u_{k}^{\prime} \\
& =\int_{\mathbb{R}^{d}}\left(\mathrm{e}^{-\psi\left(u_{1}\right)}-1\right) d u_{1} \times \int_{\left(\mathbb{R}^{d}\right)^{k-1}} U_{\mu}^{(k)}\left(u_{2}, u_{3}, \ldots, u_{k}\right) \mathrm{d} u_{2} \cdots \mathrm{d} u_{k} .
\end{aligned}
$$

Note also that

$$
|x(\varepsilon)| \rightarrow+\infty \text { and }|y(\varepsilon)| \rightarrow+\infty \text { as } \varepsilon \rightarrow 0,
$$

and hence, for each fixed $u \in \mathbb{R}^{d}$

$$
\exp [-A \phi(u-x(\varepsilon))]-1 \rightarrow 0, \quad \exp [-B \phi(u-y(\varepsilon))]-1 \rightarrow 0
$$

as $\varepsilon \rightarrow 0$. Hence, using the dominated convergence theorem, we conclude that

$$
\int_{\left(\mathbb{R}^{d}\right)^{n}}\left(f_{1, \varepsilon}^{\otimes n_{1}} \otimes f_{2, \varepsilon}^{\otimes n_{2}} \otimes f_{3, \varepsilon}^{\otimes n_{3}}\right)\left(u_{1}, \ldots, u_{n}\right) u_{\mu}\left(\eta_{1}\right) \cdots u_{\mu}\left(\eta_{j}\right) \mathrm{d} u_{1} \cdots \mathrm{d} u_{n} \rightarrow 0
$$

as $\varepsilon \rightarrow 0$.

Case 3: Case 2 is not satisfied, but there is an element $\eta_{l}$ of the partition which has non-empty intersections with both sets $\left\{u_{n_{1}+1}, \ldots, u_{n_{1}+n_{2}}\right\}$, and $\left\{u_{n_{1}+n_{2}+1}, \ldots, u_{n}\right\}$.

Shift all the variables entering $\eta_{l}$ by $x(\varepsilon)$. Now, since $\exp [-A \phi]-1 \in L^{1}\left(\mathbb{R}^{d}, d x\right)$, analogously to case 2 , the term converges to zero as $\varepsilon \rightarrow 0$.

Thus, again using the definition of the Ursell functions, we get, for each $n \in \mathbb{N}$,

$$
\begin{aligned}
C_{\varepsilon}^{(n)} & \rightarrow \sum_{n_{1}+n_{2}+n_{3}=n}\left(\begin{array}{c}
n \\
n_{1} n_{2} n_{3}
\end{array}\right) \\
& \times \int_{\left(\mathbb{R}^{d}\right)^{n_{1}}}(\exp [\psi(\cdot)]-1)^{\otimes n_{1}}\left(u_{1}, \ldots, u_{n_{1}}\right) k_{\mu}^{\left(n_{1}\right)}\left(u_{1}, \ldots, u_{n_{1}}\right) \mathrm{d} u_{1} \cdots \mathrm{d} u_{n_{1}} \\
& \times \int_{\left(\mathbb{R}^{d}\right)^{n_{2}}}(\exp [-A \phi(\cdot)]-1)^{\otimes n_{2}}\left(u_{n_{1}+1}, \ldots, u_{n_{1}+n_{2}}\right) k_{\mu}^{\left(n_{2}\right)}\left(u_{n_{1}+1}, \ldots, u_{n_{1}+n_{2}}\right) \mathrm{d} u_{n_{1}+1} \cdots \mathrm{d} u_{n_{1}+n_{2}} \\
& \times \int_{\left(\mathbb{R}^{d}\right)^{n_{3}}}(\exp [-B \phi(\cdot)]-1)^{\otimes n_{3}}\left(u_{n_{1}+n_{2}+1}, \ldots, u_{n}\right) k_{\mu}^{\left(n_{3}\right)}\left(u_{n_{1}+n_{2}+1}, \ldots, u_{n}\right) \mathrm{d} u_{n_{1}+n_{2}+1} \cdots \mathrm{d} u_{n} .
\end{aligned}
$$

Therefore, the right hand side of (9) converges to

$$
\begin{aligned}
& \left(1+\sum_{n=1}^{\infty} \frac{1}{n !} \int_{\left(\mathbb{R}^{d}\right)^{n}}(\exp [-A \phi(\cdot)]-1)^{\otimes n}\left(u_{1}, \ldots, u_{n}\right) k_{\mu}^{(n)}\left(u_{1}, \ldots, u_{n}\right) \mathrm{d} u_{1} \cdots \mathrm{d} u_{n}\right) \\
& \times\left(1+\sum_{n=1}^{\infty} \frac{1}{n !} \int_{\left(\mathbb{R}^{d}\right)^{n}}(\exp [-B \phi(\cdot)]-1)^{\otimes n}\left(u_{1}, \ldots, u_{n}\right) k_{\mu}^{(n)}\left(u_{1}, \ldots, u_{n}\right) \mathrm{d} u_{1} \cdots \mathrm{d} u_{n}\right) \\
& \times\left(1+\sum_{n=1}^{\infty} \frac{1}{n !} \int_{\left(\mathbb{R}^{d}\right)^{n}}(\exp [\psi(\cdot)]-1)^{\otimes n}\left(u_{1}, \ldots, u_{n}\right) k_{\mu}^{(n)}\left(u_{1}, \ldots, u_{n}\right) \mathrm{d} u_{1} \cdots \mathrm{d} u_{n}\right) \\
& \quad=\int_{\Gamma} \exp \left[-A \sum_{u \in \gamma} \phi(u)\right] \mu(\mathrm{d} \gamma) \int_{\Gamma} \exp \left[-B \sum_{u \in \gamma} \phi(u)\right] \mu(\mathrm{d} \gamma) \int_{\Gamma} \exp [\langle\psi, \gamma\rangle] \mu(\mathrm{d} \gamma) .
\end{aligned}
$$

as $\varepsilon \rightarrow 0$, which proves the lemma.

Now we are in position to prove the theorem. We fix any $\varphi \in C_{0}\left(\mathbb{R}^{d}\right)$ and denote $F(\gamma):=\mathrm{e}^{\langle\varphi, \gamma\rangle}$. It suffices to prove that

$$
\int_{\Gamma}\left(H_{\varepsilon}^{ \pm} F\right)^{2}(\gamma) \mu(\mathrm{d} \gamma) \rightarrow \int_{\Gamma}\left(H_{0}^{ \pm} F\right)^{2}(\gamma) \mu(\mathrm{d} \gamma) \text { as } \varepsilon \rightarrow 0,
$$




$$
\int_{\Gamma}\left(H_{\varepsilon}^{ \pm} F\right)(\gamma)\left(H_{0}^{ \pm} F\right)(\gamma) \mu(\mathrm{d} \gamma) \rightarrow \int_{\Gamma}\left(H_{0}^{ \pm} F\right)^{2}(\gamma) \mu(\mathrm{d} \gamma) \text { as } \varepsilon \rightarrow 0
$$

Now,

$$
\begin{aligned}
\int_{\Gamma} & \left.H_{0}^{-} F\right)^{2}(\gamma) \mu(\mathrm{d} \gamma) \\
= & \int_{\Gamma}\left(-\int_{\mathbb{R}^{d}} \gamma(\mathrm{d} x) \exp [s E(x, \gamma \backslash x)]\left(D_{x}^{-} F\right)(\gamma)\right)^{2} \mu(\mathrm{d} \gamma) \\
= & \int_{\Gamma} \mu(\mathrm{d} \gamma)\left(-\int_{\mathbb{R}^{d}} \gamma(\mathrm{d} x) \exp [s E(x, \gamma \backslash x)]\left(\mathrm{e}^{\langle\varphi, \gamma\rangle-\varphi(x)}-\mathrm{e}^{\langle\varphi, \gamma\rangle}\right)\right)^{2} \\
= & \int_{\Gamma} \mu(\mathrm{d} \gamma)\left(-\int_{\mathbb{R}^{d}} \gamma(\mathrm{d} x) \exp [s E(x, \gamma \backslash x)] \mathrm{e}^{\langle\varphi, \gamma\rangle}\left(\mathrm{e}^{-\varphi(x)}-1\right)\right)^{2} \\
= & \int_{\Gamma} \mu(\mathrm{d} \gamma) \mathrm{e}^{\langle 2 \varphi, \gamma\rangle} \int_{\mathbb{R}^{d}} \gamma\left(\mathrm{d} x_{1}\right) \int_{\mathbb{R}^{d}} \gamma\left(\mathrm{d} x_{2}\right) \exp \left[s E\left(x_{1}, \gamma \backslash x_{1}\right)\right] \exp \left[s E\left(x_{2}, \gamma \backslash x_{2}\right)\right] \\
& \times\left(\mathrm{e}^{-\varphi\left(x_{1}\right)}-1\right)\left(\mathrm{e}^{-\varphi\left(x_{2}\right)}-1\right) \\
= & \int_{\Gamma} \mu(\mathrm{d} \gamma) \int_{\mathbb{R}^{d}} z \mathrm{~d} x \mathrm{e}^{-E(x, \gamma)} \mathrm{e}^{\langle 2 \varphi, \gamma \cup x\rangle} \exp [2 s E(x, \gamma)]\left(\mathrm{e}^{-\varphi(x)}-1\right)^{2} \\
& +\int_{\Gamma} \mu(\mathrm{d} \gamma) \int_{\mathbb{R}^{d}} z \mathrm{~d} x_{1} \int_{\mathbb{R}^{d}} z \mathrm{~d} x_{2} \exp \left[-E\left(x_{1}, \gamma\right)-E\left(x_{2}, \gamma\right)-\phi\left(x_{1}-x_{2}\right)\right] \mathrm{e}^{\left\langle 2 \varphi, \gamma \cup x_{1} \cup x_{2}\right\rangle} \\
& \times \exp \left[s E\left(x_{1}, \gamma \cup x_{2}\right)\right] \exp \left[s E\left(x_{2}, \gamma \cup x_{1}\right)\right]\left(\mathrm{e}^{-\varphi\left(x_{1}\right)}-1\right)\left(\mathrm{e}^{-\varphi\left(x_{2}\right)}-1\right) \\
= & \int_{\mathbb{R}^{d}} z \mathrm{~d} x\left(1-\mathrm{e}^{\varphi(x)}\right)^{2} \int_{\Gamma} \mu(\mathrm{d} \gamma) \exp [(2 s-1) E(x, \gamma)+\langle 2 \varphi, \gamma\rangle] \\
& +\int_{\mathbb{R}^{d}} z \mathrm{~d} x_{1} \int_{\mathbb{R}^{d}} z \mathrm{~d} x_{2} \mathrm{e}^{\varphi\left(x_{1}\right)}\left(1-\mathrm{e}^{\varphi\left(x_{1}\right)}\right) \mathrm{e}^{\varphi\left(x_{2}\right)}\left(1-\mathrm{e}^{\varphi\left(x_{2}\right)}\right) \\
& \times \exp \left[(2 s-1) \phi\left(x_{1}-x_{2}\right)\right] \int_{\Gamma} \mu(\mathrm{d} \gamma) \exp \left[(s-1) E\left(x_{1}, \gamma\right)+(s-1) E\left(x_{2}, \gamma\right)+\langle 2 \varphi, \gamma\rangle\right] \\
& \\
& \\
&
\end{aligned}
$$

and

$$
\begin{aligned}
\int_{\Gamma}\left(H_{0}^{+} F\right)^{2}(\gamma) \mu(\mathrm{d} \gamma)= & \int_{\mathbb{R}^{d}} z \mathrm{~d} x_{1} \int_{\mathbb{R}^{d}} z \mathrm{~d} x_{2}\left(\mathrm{e}^{\varphi\left(x_{1}\right)}-1\right)\left(\mathrm{e}^{\varphi\left(x_{2}\right)}-1\right) \int_{\Gamma} \mu(\mathrm{d} \gamma) \exp \left[(s-1) E\left(x_{1}, \gamma\right)\right. \\
& \left.+(s-1) E\left(x_{2}, \gamma\right)+\langle 2 \varphi, \gamma\rangle\right] .
\end{aligned}
$$

Completely analogously to (14) we have

$$
\begin{aligned}
& \int_{\Gamma}\left(H_{\varepsilon}^{-} F\right)^{2}(\gamma) \mu(\mathrm{d} \gamma)=\int_{\mathbb{R}^{d}} z \mathrm{~d} x \int_{\mathbb{R}^{d}} \mathrm{~d} y_{1} \int_{\mathbb{R}^{d}} \mathrm{~d} y_{2}\left(\mathrm{e}^{\varphi(x)}-1\right)^{2} a_{\varepsilon}\left(x-y_{1}\right) a_{\varepsilon}\left(x-y_{2}\right) \int_{\Gamma} \mu(\mathrm{d} \gamma) \\
& \quad \times \exp \left[(2 s-1) E(x, \gamma)+(s-1) E\left(y_{1}, \gamma\right)+(s-1) E\left(y_{2}, \gamma\right)+\langle 2 \varphi, \gamma\rangle\right] \\
& \quad+\int_{\mathbb{R}^{d}} z \mathrm{~d} x_{1} \int_{\mathbb{R}^{d}} z \mathrm{~d} x_{2} \int_{\mathbb{R}^{d}} \mathrm{~d} y_{1} \int_{\mathbb{R}^{d}} \mathrm{~d} y_{2} \mathrm{e}^{\varphi\left(x_{1}\right)}\left(\mathrm{e}^{\varphi\left(x_{1}\right)}-1\right) \mathrm{e}^{\varphi\left(x_{2}\right)}\left(\mathrm{e}^{\varphi\left(x_{2}\right)}-1\right) \\
& \quad \times a_{\varepsilon}\left(x_{1}-y_{1}\right) a_{\varepsilon}\left(x_{2}-y_{2}\right) \exp \left[(2 s-1) \phi\left(x_{1}-x_{2}\right)+(s-1) \phi\left(y_{1}-x_{2}\right)\right. \\
& \left.\quad+(s-1) \phi\left(x_{1}-y_{2}\right)\right] \int_{\Gamma} \mu(\mathrm{d} \gamma) \exp \left[(s-1) E\left(x_{1}, \gamma\right)+(s-1) E\left(x_{2}, \gamma\right)\right. \\
& \left.\quad+(s-1) E\left(y_{1}, \gamma\right)+(s-1) E\left(y_{2}, \gamma\right)+\langle 2 \varphi, \gamma\rangle\right] .
\end{aligned}
$$

Let us make the change of variables

$$
y_{1}^{\prime}=\varepsilon\left(y_{1}-x\right), \quad y_{2}^{\prime}=\varepsilon\left(y_{2}-x\right)
$$


in the first integral, and

$$
y_{1}^{\prime}=\varepsilon\left(y_{1}-x_{1}\right), \quad y_{2}^{\prime}=\varepsilon\left(y_{2}-x_{2}\right)
$$

in the second integral. Then, omitting the primes in the notations of variables, we continue (16) as follows:

$$
\begin{aligned}
= & \int_{\mathbb{R}^{d}} z \mathrm{~d} x \int_{\mathbb{R}^{d}} \mathrm{~d} y_{1} \int_{\mathbb{R}^{d}} \mathrm{~d} y_{2}\left(\mathrm{e}^{\varphi(x)}-1\right)^{2} a\left(y_{1}\right) a\left(y_{2}\right) \int_{\Gamma} \mu(\mathrm{d} \gamma) \exp [(2 s-1) E(x, \gamma) \\
& \left.+(s-1) E\left(\left(y_{1} / \varepsilon\right)+x, \gamma\right)+(s-1) E\left(\left(y_{2} / \varepsilon\right)+x, \gamma\right)+\langle 2 \varphi, \gamma\rangle\right] \\
& +\int_{\mathbb{R}^{d}} z \mathrm{~d} x_{1} \int_{\mathbb{R}^{d}} z \mathrm{~d} x_{2} \int_{\mathbb{R}^{d}} \mathrm{~d} y_{1} \int_{\mathbb{R}^{d}} \mathrm{~d} y_{2} \mathrm{e}^{\varphi\left(x_{1}\right)}\left(\mathrm{e}^{\varphi\left(x_{1}\right)}-1\right) \mathrm{e}^{\varphi\left(x_{2}\right)}\left(\mathrm{e}^{\varphi\left(x_{2}\right)}-1\right) \\
& \times a\left(y_{1}\right) a\left(y_{2}\right) \exp \left[(2 s-1) \phi\left(x_{1}-x_{2}\right)+(s-1) \phi\left(\left(y_{1} / \varepsilon\right)+x_{1}-x_{2}\right)\right. \\
& +(s-1) \phi\left(\left(y_{2} / \varepsilon\right)+x_{2}-x_{1}\right) \int_{\Gamma} \mu(\mathrm{d} \gamma) \exp \left[(s-1) E\left(x_{1}, \gamma\right)+(s-1) E\left(x_{2}, \gamma\right)\right. \\
& \left.+(s-1) E\left(\left(y_{1} / \varepsilon\right)+x_{1}, \gamma\right)+(s-1) E\left(\left(y_{2} / \varepsilon\right)+x_{2}, \gamma\right)+\langle 2 \varphi, \gamma\rangle\right] .
\end{aligned}
$$

Next,

$$
\begin{aligned}
\int_{\Gamma} & \left.H_{\varepsilon}^{+} F\right)^{2}(\gamma) \mu(\mathrm{d} \gamma) \\
= & \int_{\Gamma} \mu(\mathrm{d} \gamma)\left(-\int_{\mathbb{R}^{d}} \gamma(\mathrm{d} x) \int_{\mathbb{R}^{d}} \mathrm{~d} y a_{\varepsilon}(x-y)\right. \\
& \times \exp [s E(x, \gamma \backslash x)-(1-s) E(y, \gamma \backslash x)](F(\gamma \backslash x \cup y)-F(\gamma \backslash x)))^{2} \\
= & \int_{\Gamma} \mu(\mathrm{d} \gamma)\left(\int_{\mathbb{R}^{d}} \gamma(\mathrm{d} x) \int_{\mathbb{R}^{d}} \mathrm{~d} y a_{\varepsilon}(x-y)\right. \\
& \left.\times \exp [s E(x, \gamma \backslash x)-(1-s) E(y, \gamma \backslash x)]\left(\mathrm{e}^{\langle\varphi, \gamma \backslash x\rangle+\varphi(y)}-\mathrm{e}^{\langle\varphi, \gamma \backslash x\rangle}\right)\right)^{2} \\
= & \int_{\Gamma} \mu(\mathrm{d} \gamma)\left(\int_{\mathbb{R}^{d}} \gamma\left(\mathrm{d} x_{1}\right) \int_{\mathbb{R}^{d}} \gamma\left(\mathrm{d} x_{2}\right) \mathrm{e}^{\left\langle\varphi, \gamma \backslash x_{1}\right\rangle} \mathrm{e}^{\left\langle\varphi, \gamma \backslash x_{2}\right\rangle} \int_{\mathbb{R}^{d}} \mathrm{~d} y_{1} \int_{\mathbb{R}^{d}} \mathrm{~d} y_{2}\right. \\
& \times a_{\varepsilon}\left(x_{1}-y_{1}\right) a_{\varepsilon}\left(x_{2}-y_{2}\right) \exp \left[s E\left(x_{1}, \gamma \backslash x_{1}\right)-(1-s) E\left(y_{1}, \gamma \backslash x_{1}\right)\right] \\
& \times \exp \left[s E\left(x_{2}, \gamma \backslash x_{2}\right)-(1-s) E\left(y_{2}, \gamma \backslash x_{2}\right)\right]\left(\mathrm{e}^{\varphi\left(y_{1}\right)}-1\right)\left(\mathrm{e}^{\varphi\left(y_{2}\right)}-1\right) \\
= & \int_{\Gamma} \mu(\mathrm{d} \gamma) \int_{\mathbb{R}^{d}} \gamma(\mathrm{d} x) \mathrm{e}^{\langle 2 \varphi, \gamma \backslash x\rangle} \int_{\mathbb{R}^{d}} \mathrm{~d} y_{1} \int_{\mathbb{R}^{d}} \mathrm{~d} y_{2} \\
& \times a_{\varepsilon}\left(x-y_{1}\right) a_{\varepsilon}\left(x-y_{2}\right) \exp \left[s E(x, \gamma \backslash x)-(1-s) E\left(y_{1}, \gamma \backslash x\right)\right] \\
& \times \exp \left[s E(x, \gamma \backslash x)-(1-s) E\left(y_{2}, \gamma \backslash x\right)\right]\left(\mathrm{e}^{\varphi\left(y_{1}\right)}-1\right)\left(\mathrm{e}^{\varphi\left(y_{2}\right)}-1\right) \\
& +\int_{\Gamma} \mu(\mathrm{d} \gamma) \int_{\mathbb{R}^{d}} \gamma\left(\mathrm{d} x_{1}\right) \int_{\mathbb{R}^{d}}\left(\gamma \backslash x_{1}\right)\left(\mathrm{d} x_{2}\right) \mathrm{e}^{\left\langle\varphi, \gamma \backslash x_{1}\right\rangle} \mathrm{e}^{\left\langle\varphi, \gamma \backslash x_{2}\right\rangle} \int_{\mathbb{R}^{d}} \mathrm{~d} y_{1} \int_{\mathbb{R}^{d}} \mathrm{~d} y_{2} \\
& \times a_{\varepsilon}\left(x_{1}-y_{1}\right) a_{\varepsilon}\left(x_{2}-y_{2}\right) \exp \left[s E\left(x_{1}, \gamma \backslash x_{1}\right)-(1-s) E\left(y_{1}, \gamma \backslash x_{1}\right)\right] \\
& \times \exp \left[s E\left(x_{2}, \gamma \backslash x_{2}\right)-(1-s) E\left(y_{2}, \gamma \backslash x_{2}\right)\right]\left(\mathrm{e}^{\varphi\left(y_{1}\right)}-1\right)\left(\mathrm{e}^{\varphi\left(y_{2}\right)}-1\right) \\
& \times \exp \left[s E(x, \gamma)-(1-s) E\left(y_{2}, \gamma\right)\right]\left(\mathrm{e}^{\varphi\left(y_{1}\right)}-1\right)\left(\mathrm{e}^{\varphi\left(y_{2}\right)}-1\right) \\
& \int_{\mathbb{R}^{d}}[(x, \gamma)] \mathrm{e}^{\langle 2 \varphi, \gamma\rangle} \int_{\mathbb{R}^{d}} \mathrm{~d} y_{1} \int_{\mathbb{R}^{d}} \mathrm{~d} y_{2} \\
& \\
& \\
&
\end{aligned}
$$




$$
\begin{aligned}
& +\int_{\Gamma} \mu(\mathrm{d} \gamma) \int_{\mathbb{R}^{d}} z \mathrm{~d} x_{1} \int_{\mathbb{R}^{d}} z \mathrm{~d} x_{2} \int_{\mathbb{R}^{d}} \mathrm{~d} y_{1} \int_{\mathbb{R}^{d}} \mathrm{~d} y_{2} \\
& \times \exp \left[-E\left(x_{1}, \gamma\right)-E\left(x_{2}, \gamma\right)-\phi\left(x_{1}-x_{2}\right)\right] \mathrm{e}^{\left\langle\varphi, \gamma \cup x_{2}\right\rangle} \mathrm{e}^{\left\langle\varphi, \gamma \cup x_{1}\right\rangle} \\
& \times a_{\varepsilon}\left(x_{1}-y_{1}\right) a_{\varepsilon}\left(x_{2}-y_{2}\right) \exp \left[s E\left(x_{1}, \gamma \cup x_{2}\right)-(1-s) E\left(y_{1}, \gamma \cup x_{2}\right)\right] \\
& \times \exp \left[s E\left(x_{2}, \gamma \cup x_{1}\right)-(1-s) E\left(y_{2}, \gamma \cup x_{1}\right)\right]\left(\mathrm{e}^{\varphi\left(y_{1}\right)}-1\right)\left(\mathrm{e}^{\varphi\left(y_{2}\right)}-1\right) \\
& =: \mathrm{I}+\mathrm{II} .
\end{aligned}
$$

In the first integral in (18) let us make the change of variables

$$
y_{1}^{\prime}=\varepsilon\left(y_{1}-x\right), \quad y_{2}^{\prime}=\varepsilon\left(y_{2}-x\right) .
$$

Then, omitting the primes in the notations of variables, we continue I as follows:

$$
\begin{aligned}
\mathrm{I}= & \int_{\mathbb{R}^{d}} \mathrm{~d} y_{1} \int_{\mathbb{R}^{d}} \mathrm{~d} y_{2} a\left(y_{1}\right) a\left(y_{2}\right)\left(\mathrm{e}^{\varphi\left(\left(y_{1} / \varepsilon\right)+x\right)}-1\right)\left(\mathrm{e}^{\varphi\left(\left(y_{2} / \varepsilon\right)+x\right)}-1\right) \\
& \times \int_{\Gamma} \mu(\mathrm{d} \gamma) \int_{\mathbb{R}^{d}} z \mathrm{~d} x \exp \left[(2 s-1) E(x, \gamma)+(s-1) E\left(\left(y_{1} / \varepsilon\right)+x, \gamma\right)\right. \\
& \left.+(s-1) E\left(\left(y_{2} / \varepsilon\right)+x, \gamma\right)+\langle 2 \varphi, \gamma\rangle\right] .
\end{aligned}
$$

Let us take $x^{\prime}=x+\left(y_{1} / \varepsilon\right)$, then omitting the primes in the notations of variables, we get:

$$
\begin{aligned}
\mathrm{I}= & \int_{\mathbb{R}^{d}} \mathrm{~d} y_{1} \int_{\mathbb{R}^{d}} \mathrm{~d} y_{2} a\left(y_{1}\right) a\left(y_{2}\right) \int_{\mathbb{R}^{d}} z \mathrm{~d} x\left(\mathrm{e}^{\varphi(x)}-1\right)\left(\mathrm{e}^{\varphi\left(x+\left(\left(y_{2}-y_{1}\right) / \varepsilon\right)\right)}-1\right) a\left(y_{1}\right) a\left(y_{2}\right) \\
& \times \int_{\Gamma} \mu(\mathrm{d} \gamma) \exp \left[(2 s-1) E\left(x-\left(y_{1} / \varepsilon\right), \gamma\right)\right. \\
& \left.+(s-1) E(x, \gamma)+(s-1) E\left(x+\left(\left(y_{2}-y_{1}\right) / \varepsilon\right), \gamma\right)+\langle 2 \varphi, \gamma\rangle\right] .
\end{aligned}
$$

In the second integral in (18), let us make the change of variables $x_{1}^{\prime}=\varepsilon\left(x_{1}-y_{1}\right), x_{2}^{\prime}=\varepsilon\left(x_{2}-y_{2}\right)$. Then omitting the primes, we have:

$$
\begin{aligned}
\mathrm{II}= & \int_{\mathbb{R}^{d}} z \mathrm{~d} x_{1} \int_{\mathbb{R}^{d}} z \mathrm{~d} x_{2} \int_{\mathbb{R}^{d}} \mathrm{~d} y_{1} \int_{\mathbb{R}^{d}} \mathrm{~d} y_{2} \mathrm{e}^{\varphi\left(\left(x_{1} / \varepsilon\right)+y_{1}\right)} \mathrm{e}^{\varphi\left(\left(x_{2} / \varepsilon\right)+y_{2}\right)}\left(\mathrm{e}^{\varphi\left(y_{1}\right)}-1\right)\left(\mathrm{e}^{\varphi\left(y_{2}\right)}-1\right) \\
& \times a\left(x_{1}\right) a\left(x_{2}\right) \exp \left[(2 s-1) \phi\left(\left(\left(x_{1}-x_{2}\right) / \varepsilon\right)+y_{1}-y_{2}\right)+(s-1) \phi\left(\left(x_{1} / \varepsilon\right)+y_{1}-y_{2}\right)\right. \\
& \left.+(s-1) \phi\left(y_{1}-y_{2}-\left(x_{2} / \varepsilon\right)\right)\right] \int_{\Gamma} \mu(\mathrm{d} \gamma) \exp \left[(s-1) E\left(y_{1}, \gamma\right)+(s-1) E\left(y_{2}, \gamma\right)\right. \\
& \left.+(s-1) E\left(\left(x_{1} / \varepsilon\right)+y_{1}, \gamma\right)+(s-1) E\left(\left(x_{2} / \varepsilon\right)+y_{2}, \gamma\right)+\langle 2 \varphi, \gamma\rangle\right] .
\end{aligned}
$$

Using (19) and (20), we get

$$
\begin{aligned}
& \int_{\Gamma}\left(H_{\varepsilon}^{+} F\right)^{2}(\gamma) \mu(\mathrm{d} \gamma)=\int_{\mathbb{R}^{d}} z \mathrm{~d} x \int_{\mathbb{R}^{d}} \mathrm{~d} y_{1} \int_{\mathbb{R}^{d}} \mathrm{~d} y_{2}\left(\mathrm{e}^{\varphi(x)}-1\right)\left(\mathrm{e}^{\varphi\left(x+\left(\left(y_{2}-y_{1}\right) / \varepsilon\right)\right.}-1\right) a\left(y_{1}\right) a\left(y_{2}\right) \\
& \quad \times \int_{\Gamma} \mu(\mathrm{d} \gamma) \exp \left[(2 s-1) E\left(x-\left(y_{1} / \varepsilon\right), \gamma\right)\right. \\
& \left.\quad+(s-1) E(x, \gamma)+(s-1) E\left(x+\left(\left(y_{2}-y_{1}\right) / \varepsilon\right), \gamma\right)+\langle 2 \varphi, \gamma\rangle\right] \\
& \quad+\int_{\mathbb{R}^{d}} z \mathrm{~d} x_{1} \int_{\mathbb{R}^{d}} z \mathrm{~d} x_{2} \int_{\mathbb{R}^{d}} \mathrm{~d} y_{1} \int_{\mathbb{R}^{d}} \mathrm{~d} y_{2} \mathrm{e}^{\varphi\left(\left(x_{1} / \varepsilon\right)+y_{1}\right)} \mathrm{e}^{\varphi\left(\left(x_{2} / \varepsilon\right)+y_{2}\right)}\left(\mathrm{e}^{\varphi\left(y_{1}\right)}-1\right)\left(\mathrm{e}^{\varphi\left(y_{2}\right)}-1\right) \\
& \quad \times a\left(x_{1}\right) a\left(x_{2}\right) \exp \left[(2 s-1) \phi\left(\left(\left(x_{1}-x_{2}\right) / \varepsilon\right)+y_{1}-y_{2}\right)+(s-1) \phi\left(\left(x_{1} / \varepsilon\right)+y_{1}-y_{2}\right)\right. \\
& \quad+(s-1) \phi\left(y_{1}-y_{2}-\left(x_{2} / \varepsilon\right)\right) \int_{\Gamma} \mu(\mathrm{d} \gamma) \exp \left[(s-1) E\left(y_{1}, \gamma\right)+(s-1) E\left(y_{2}, \gamma\right)\right. \\
& \left.\quad+(s-1) E\left(\left(x_{1} / \varepsilon\right)+y_{1}, \gamma\right)+(s-1) E\left(\left(x_{2} / \varepsilon\right)+y_{2}, \gamma\right)+\langle 2 \varphi, \gamma\rangle\right] .
\end{aligned}
$$


Completely analogously, we get

$$
\begin{aligned}
& \int_{\Gamma}\left(H_{0}^{-} F\right)(\gamma)\left(H_{\varepsilon}^{-} F\right)(\gamma) \mu(\mathrm{d} \gamma) \\
& =\int_{\mathbb{R}^{d}} z \mathrm{~d} x \int_{\mathbb{R}^{d}} \mathrm{~d} y\left(\mathrm{e}^{\varphi(x)}-1\right)^{2} a(y) \int_{\Gamma} \mu(\mathrm{d} \gamma) \exp [(2 s-1) E(x, \gamma)+(s-1) E((y / \varepsilon)+x, \gamma)+\langle 2 \varphi, \gamma\rangle] \\
& \quad+\int_{\mathbb{R}^{d}} z \mathrm{~d} x_{1} \int_{\mathbb{R}^{d}} z \mathrm{~d} x_{2} \int_{\mathbb{R}^{d}} \mathrm{~d} y\left(\mathrm{e}^{\varphi\left(x_{1}\right)}-1\right)\left(\mathrm{e}^{\varphi\left(x_{2}\right)}-1\right) \mathrm{e}^{\varphi\left(x_{1}\right)} \mathrm{e}^{\varphi\left(x_{2}\right)} a(y) \\
& \quad \times \exp \left[(2 s-1) \phi\left(x_{1}-x_{2}\right)+(s-1) \phi\left((y / \varepsilon)+x_{1}-x_{2}\right)\right] \\
& \quad \times \int_{\Gamma} \mu(\mathrm{d} \gamma) \exp \left[(s-1) E\left(x_{1}, \gamma\right)+(s-1) E\left(x_{2}, \gamma\right)+(s-1) E\left((y / \varepsilon)+x_{1}, \gamma\right)+\langle 2 \varphi, \gamma\rangle\right]
\end{aligned}
$$

and

$$
\begin{aligned}
& \int_{\Gamma}\left(H_{0}^{+} F\right)(\gamma)\left(H_{\varepsilon}^{+} F\right)(\gamma) \mu(\mathrm{d} \gamma)=\int_{\mathbb{R}^{d}} z \mathrm{~d} x_{1} \int_{\mathbb{R}^{d}} z \mathrm{~d} x_{2} \int_{\mathbb{R}^{d}} \mathrm{~d} y a\left(x_{2}\right)\left(\mathrm{e}^{\varphi\left(x_{1}\right)}-1\right) \\
& \quad \times\left(\mathrm{e}^{\varphi(y)}-1\right) \int_{\Gamma} \mu(\mathrm{d} \gamma) \mathrm{e}^{\langle 2 \varphi, \gamma\rangle} \exp \left[(s-1) E\left(\left(x_{2} / \varepsilon\right)+y, \gamma\right)+(s-1) E(y, \gamma)\right. \\
& \left.\quad+(s-1) E\left(x_{1}, \gamma\right)+(s-1) \phi\left(\left(x_{2} / \varepsilon\right)+y-x_{1}\right)\right] .
\end{aligned}
$$

Using the Ruelle bound and Lemma 1, we conclude that the integral over $\Gamma$ in the right hand side of equalities $(17),(21)-(23)$, is bounded by a constant, which is independent of $\varepsilon$. Therefore, by the dominated convergence theorem, to find the limit of (17), (21)-(23) as $\varepsilon \rightarrow 0$ it suffices to

find the point-wise limit of the functions appearing before the integral over $\Gamma$, as well as the limit of the integrals over $\Gamma$ for fixed $x$ ( $x_{1}$ and $x_{2}$ respectively), $y_{1}$ and $y_{2}$.

To find the latter limits, we use Lemms 3. Then, using (8), we see that (17) and (22) converge to (14), whereas (21) and (23) converge to (15). Therefore, (12) and (13) hold.

\section{References}

1. Bertini L., Cancrini N., Cesi F., The spectral gap for a Glauber-type dynamics in a continuous gas. Ann. Inst. H. Poincaré Probab. Statist., 2002, 38, 91.

2. Alberverio S., Kondratiev Y.G., Röckner M., Analysis and geometry on configuration spaces. The Gibbsian case. J. Funct. Anal., 1998, 157, 242.

3. Finkelshtein D.L., Kondratiev Y.G., Lytvynov E.W., Glauber dynamics as a scaling limit of Kawasaki dynamics. Random Oper. Stoch. Equ., 2007, 15, 105.

4. Fritz J., Gradient stochastic dynamics of infinite particle systems. Ann. Prob., 1987, 15, 478.

5. Fukushima M., Dirichlet Forms and Symmetric Markov Processes. North-Holland, Amsterdam, New York, 1980.

6. Glötzl E., Time reversible and Gibbsian point processes. I. Markovian spatial birth and death processes on a general phase space. Math. Nachr., 1981, 102, 217.

7. Glötzl E., Time reversible and Gibbsian point processes. II. Markovian particle jump processes on a general phase space. Math. Nachr., 1982, 106, 63.

8. Holley R.A., Stroock D.W., Nearest neighbor birth and death processes on the real line. Acta Math., 1987, 140, 103.

9. Kallenberg O., Random Measures. Akademie-Verlag, Berlin; Academic Press, London, New York, 1976.

10. Kondratiev Y.G., Kutiviy O.L., Lytvynov E.W., Diffusion approximation for equilibrium Kawasaki dynamics in continuum. Stochastic Process. Appl., 2008, 118, 1278.

11. Kondratiev Y.G., Lytvynov E., Glauber dynamics of continuous particle systems. Ann. Inst. H. Poincaré Probab., Statist., 2005, 41, 685.

12. Kondratiev Y.G., Lytvynov E., Röckner M., Infinite interacting diffusion particles I: Equilibrium process and its scaling limit. Forum Math., 2006, 18, 9.

13. Kondratiev Y.G., Lytvynov E., Röckner M., Equilibrium Kawasaki dynamics of continuous particle systems. Infin. Dimens. Anal. Quantum Probab. Relat. Top., 2007, 10, 185.

14. Kondratiev Y., Minlos R., Zhizhina E., One-particle subspace of the Glauber dynamics generator for continuous particle systems. Rev. Math. Phys., 2004, 16, 1073. 
15. Ma Z.-M., Röckner M., An Introduction to the Theory of (Non-Symmetric) Dirichlet Forms. SpringerVerlag, Berlin, 1992.

16. Ma Z.-M., Röckner M., Construction of diffusions on configuration spaces. Osaka J. Math., 2000, 37, 273.

17. Nguyen X.X., Zessin H., Integral and differentiable characterizations of the Gibbs process. Math. Nachr., 1979, 88, 105.

18. Osada H., Dirichlet form approach to infinite-dimensional Wiener processes with singular interaction. Comm. Math. Phys., 1996, 176, 117.

19. Preston C., Spatial birth-and-death processes. - In: Proceedings of the 40th Session of the International Statistical Institute, Warsaw, 1975, 2, Bull. Inst. Internat. Statist., 46, 1975, 371-391.

20. Röckner M., Schmuland B., A support property for infinite-dimensional interacting diffusion processes. C. R. Acad. Sci. Paris, 1998, 326, Série I, 359.

21. Ruelle D., Cluster property of the correlation functions of classical gases. Rev. Mod. Phys., 1964, 36, 580 .

22. Ruelle D., Statistical Mechanics. Rigorous Results. Benjamins, New York, Amsterdam, 1969.

23. Wu L., Estimate of spectral gap for continuous gas. Ann. Inst. H. Poincaré Probab. Statist., 2004, 40, 387.

24. Yoshida M.W., Construction of infinite-dimensional interacting diffusion processes through Dirichlet forms. Prob. Theory Related Fields, 1996, 106, 265.

\title{
До збіжності генераторів рівноважної динаміки перестрибуючих частинок до генератора процесу народження-знищення в континуумі
}

\author{
Є.Литвинов, П.Т.Поляра \\ Факультет математики, Університет Суонсі, Суонсі, Великобританія \\ Отримано 31 січня 2008 р.
}

\begin{abstract}
Ми розглядаємо такі два типи рівноважних стохастичних динамік нескінченно-частинкових систем в континуумі: перестрибуючі частинки (динаміка Кавасакі), тобто динаміка, коли кожна частинка випадковим чином перескакує в просторі; динаміка типу народження-знищення (динаміка Глаубера), при якій частинки не рухаються, а народжуються і знищуються випадковим чином. Ми доводимо, що для широкого класу динамік Глаубера кожна така динаміка може бути одержана як скейлінгова границя динаміки Кавасакі. Точніше, ми доводимо збіжність відповідних генераторів на множині циліндричних функцій в нормі $L^{2}$ відносно відповідної інваріантної міри процесу. Остання є мірою Гіббса, що відповідає потенціалу парної взаємодії в режимі мала активність / високі температури. Наш результат узагальнює результат роботи [Finkelshtein D.L. et al., Random Oper. Stochastic Equations], одержаний для спеціальних типів динамік Глаубера і Кавасакі.
\end{abstract}

Ключові слова: процес народження і знищення, скінченні системи, міра Гіббса, перестрибуючі частинки, скейлінгова границя

PACS: 02.50.Ey, 02.50.Ga 